\title{
Vorwort der ersten Auflage
}

Es liegt im Wesen der deutschen wie jeder anderen Literaturgeschichte, daß sie zunächst individualistisch gerichtet ist. Das Dichtwerk als Leistung und Ausdruck einer schöpferischen Persönlichkeit und die einzelne Künstlergestalt bieten sich der forschenden und darstellenden Wissenschaft als nächstliegende Gegenstände an. In diesem Sinne kommen auch die älteren Literaturgeschichten im wesentlichen nicht viel über aneinandergereihte Einzelcharakteristiken von Kunstwerken und Dichtern hinaus. Nur in den literaturgeschichtlichen Hilfsdisziplinen der Metrik, Stilistik und Poetik standen begreiflicherweise die sachlichen Gesichtspunkte von vornherein im Vordergrunde.

Die allgemeine Wissenschaftsumstellung der beiden letzten Jahrzehnte, die bei aller bleibenden und selbstverständlichen Wertschätzung des persönlichen Moments überall einen starken Zug zum Überpersönlichen, Typischen, Allgemeinen, Grundsätzlichen erkennen ließ und neben der literarischen Kunstgeschichte die geistesgeschichtliche Literaturwissenschaft zur vollen Entfaltung führte, hat jenes sachliche Element zu ungleich stärkerer Bedeutung gebracht. Das literarische Leben erscheint nicht mehr bloß als Wirkungsfeld schaffender und gestaltender Persönlichkeiten, sondern gleichzeitig als Offenbarung tieferliegender Strömungen, Richtungen, Stilmoden, Geschmacksveränderungen. Die früher nur mehr gelegentlich und vereinzelt verfolgte Entwicklung der literarischen Formen, Gattungen, Arten, Modeerscheinungen ist damit stark in den Vordergrund des Interesses getreten. Einzelne Sachgebiete, besonders die Theatergeschichte, haben sich zu selbständiger wissenschaftlicher Bedeutung durchgerungen. Überall wird die Macht der allgemeinen Strömungen und Stimmungen deutlich, drängt die literaturwissenschaftliche Betrachtung zur Verfolgung von Längsschnitten und durchgehenden Entwicklungslinien, gliedert sich das Persönliche und Einzelne in höhere geistes- und bildungsgeschichtliche Wellenbewegungen ein. Damit aber sind die Realien der Literaturgeschichte, d. i. die Gesamtheit der über- und unterpersönlichen Faktoren, ungleich mehr als früher Gegenstand der Forschung und des Interesses geworden.

In diesem Sinne sucht das vorliegende, auf drei Bände berechnete Reallexikon der deutschen Literaturgeschichte erstmalig den sach- und formgeschichtlichen Gesichtspunkt zum herrschenden Prinzip zu erheben. Die Einzelpersönlichkeiten und ihre künstlerische Eigenart werden nur insofern Beachtung finden, als sie bei der Darstellung der sachlichen Entwicklungslinien von Bedeutung sind. Nur in der übergeordneten Form gewisser geistesgeschichtlicher und literarhistorischer Gruppenbildungen wird das personale Element stärker mitzusprechen haben. Im übrigen werden die etwa 800 Artikel dieses Lexikons die literaturwissenschaftliche Materie grundsätzlich von sachlicher und formgeschichtlicher Einstellung aus behandeln. Im einzelnen lagen für diese erst neuerdings in ihrer Eigenwertigkeit stärker beachtete „realistische“ Literaturgeschichte die Grundlagen sehr verschieden. Bei zahlreichen Artikeln konnten die Bearbeiter sich auf gute Vorarbeiten stützen; aber bei vielen anderen, oft recht bedeutsamen, galt es, durch das 
üppig wuchernde Feld der Einzelerscheinungen erstmalig eine Entwicklungsbahn zu schlagen und künftiger Forschung die Wege zu weisen. Schon dies bedingte, abgesehen von der Verschiedenheit der nahezu 100 Mitarbeiter, hier und da eine nicht zu vermeidende Ungleichheit in der Behandlungsweise, Anlagehöhe und Ausdehnung der Stichwortartikel.

Die Vorgeschichte dieses Reallexikons der deutschen Literaturgeschichte geht weit zurück und führt bis an die Schwelle der modernen geisteswissenschaftlichen Literaturwissenschaft. Bereits in demselben Jahre 1911, das in Ungers Hamannwerk und Gundolfs Shakespearebuch die ersten deutlichen Zeugen der methodischen Schwenkung brachte, entwickelte der eine der beiden Herausgeber (Prof. Merker) dem Vertreter des damaligen Trübnerschen Verlags an Hand der aufgestellten Stichworte eingehend den Plan des Unternehmens, das im ganzen betrachtet jetzt in derselben Form zur Verwirklichung gekommen ist. Obwohl der Gedanke mit einem entsprechenden Plane des Verlags zusammentraf, der seine Grundrißreihe durch eine lexikalisch eingerichtete Serie ergänzen wollte, blieb das in Aussicht genommene alphabetische Nachschlagewerk damals, durch persönliche und zeitliche Verhältnisse bedingt, im Keimstadium geplanter Entwicklung stecken. Die Kriegs- und ersten Nachkriegsjahre brachten dann aus inneren und äußeren Gründen weitere Hemmung, bis erst im Jahre 1920 eine neue, diesmal vom Verlage ausgehende Anregung das Vorhaben wiederum in Fluß brachte. Mit der wenig später erfolgten Gewinnung von Prof. Stammler als Mitherausgeber, der auch die erste Stichwortliste mannigfach ergänzte, erhielt das Unternehmen eine breitere Basis. Etwa gleichzeitig konnte an die Auswahl und Werbung der Mitarbeiter gegangen werden, die im einzelnen nicht immer leicht zu gewinnen waren (einzelne, besonders undankbare Stichworte konnten erst nach 6-8maligem Ausbieten ihren willigen Bearbeiter finden). Die unklare Lage der folgenden Inflationszeit, die nicht nur dem Verlag jede Übersichtsmöglichkeit nahm, sondern auch in den Reihen der Mitarbeiter vielfach Zweifel und Aufschubwünsche wach werden ließ, und die Notwendigkeit mannigfacher Neuwerbungen verzögerten weiterhin den Druckbeginn. Erst Anfang dieses Jahres waren die Manuskripte so weit eingelaufen, $\mathrm{da} ß$ an die Drucklegung gegangen und im Juni die erste Lieferung ausgegeben werden konnte....

Schon jetzt sei darauf hingewiesen, daß Herausgeber und Verlag den Plan verfolgen, diesem vorwiegend formgeschichtlich gerichteten Reallexikon später ein Personallexikon sowie ein Stoff- und Motivlexikon zur Seite treten zu lassen. Die drei Wurzeln und Elemente der literarischen Erscheinungen (Persönlichkeit, Stoff, Form) würden dann in drei sich ergänzenden lexikalischen Nachschlagewerken nebeneinander Berücksichtigung finden. Möge zunächst dieses „Real“-Lexikon, das wir besonders gern auch in den Händen der Studierenden und in den Schulbibliotheken sehen würden, seinen Weg gehen.

Greifswald, 30. Dezember 1925

Paul Merker

Wolfgang Stammler 